\title{
Artículos
}

\section{Impacto territorial del manejo forestal comunitario en la Sierra Norte de} Puebla, 2002-20I7*

Cómo citar este artículo: Ceballos Pérez, S. G. (2020). Impacto territorial del manejo forestal comunitario en la Sierra Norte de Puebla, $2003-2017$. Cuadernos de Desarrollo Rural, I7(86). https://doi.org/I0.III44/Javeriana.cdrı7.itmf

\section{Sergio Gabriel Ceballos Pérez a}

El Colegio del Estado de Hidalgo, México

sceballos@elcolegiodehidalgo.edu.mx

DOI: https://doi.org/IO.III44/Javeriana.cdrı7.itmf

Recibido: 22 Mayo 2019 I Aceptado: 10 Julio 2020 I Publicación: 20 Diciembre 2020

\section{Resumen:}

El objetivo de este artículo es conocer el impacto territorial que tiene el manejo forestal comunitario (MFC) en la Sierra Norte de Puebla (SNP), en el periodo que comprende del 2003 al 2017. El MFC es entendido teóricamente aquí como una forma de organización colectiva y de gobernanza ambiental; el territorio, como un reflejo del comportamiento social. En el presente texto se utiliza metodología cuantitativa, mediante el análisis de indicadores económico-ambientales, tales como volúmenes de la producción, planes de manejo, subsidios, cambios de uso del suelo; así mismo, se realizaron visitas en campo y entrevistas para el análisis cualitativo. A partir del análisis de los resultados obtenidos, pudo concluirse que la cobertura forestal no sufrió pérdidas después del MFC, por el contrario, se incrementó en un $8 \%$ (64.33 km.). Como conclusión, podría estipularse que el modelo del MFC en la SNP presenta impactos positivos sobre el territorio.

Palabras clave: Manejo forestal comunitario, Territorio, Desarrollo sustentable, Desarrollo regional, Cambios de uso de suelo.

\section{Territorial Impact of the Community Forest Management in the Sierra Norte de Puebla, 2002-20I7}

\begin{abstract}
:
This article aims to know the territorial impact of the community forest management (CFM) in the Sierra Norte de Puebla (SNP) during the term 2003-2017. The CFM is understood theoretically as a way of collective organization and environmental governance. Territory is understood as a reflection of the social behavior. This work uses a quantitative methodology based on analyzing the economic-environmental indicators,
\end{abstract}

a Autor de correspondencia. Correo electrónico: sceballos@elcolegiodehidalgo.edu.mx 
such as production volumes, management plans, subsidies, land use changes. Likewise, field visits and interviews were carried out for the qualitative analysis. Having analyzed the results, it is concluded that the forest cover did not undergo any loss after the CFM. Quite the opposite, it increased in $8 \%(64.33 \mathrm{Km}$.). As a conclusion, it could be said that the CFM model applied in the SNP has had positive impacts on the territory.

Keywords: community forest management, territory, sustainable development, regional development, land use changes.

\section{Introducción}

La relación que existe entre el territorio y el desarrollo sustentable ha sido un tema de investigación relevante en los últimos años y puede percibirse que las formas de aprovechamiento, tales como el manejo forestal comunitario (MFC), juegan un papel fundamental. Autores como McKean y Ostrom (1995), García y Antinori (20r8), Fuente y Carcaño (2016), Valdés y Negreros (2010), Bray y Merino (2005) han ahondado en el análisis de este tipo de aprovechamiento, en el que las relaciones comunitarias son la clave, ya que brindan cohesión social, capital social, autodeterminación, derechos de propiedad e incluso construcción de modelos y asociaciones económicas sui generis.

La literatura que aborda el MFC, actualmente, es muy amplia: en una búsqueda bibliográfica en los portales académicos y de revistas, tales como Researchgate, Academia, Redalyc y Elsevier, encontramos 76 artículos sin repetirse, utilizando únicamente las palabras manejo forestal comunitario y silvicultura comunitaria. La mayor parte de esta literatura se enfoca en resaltar los beneficios que trae este tipo de manejo, analizando experiencias de comunidades en países como México, Guatemala, Bolivia, Brasil y Perú. Un aspecto que se debe resaltar de estos artículos, a partir de su revisión, es que por su característica de participación social y por sus reglas de uso común han demostrado tener la capacidad de desencadenar procesos de desarrollo endógeno territorial, en el ámbito local y regional (Bray et al., 2007). En ese sentido el MFC, es una forma de manejo participativa y sustentable del territorio, por lo cual, en el manejo forestal comunitario, lo que hay que destacar es la forma de organización y las reglas que lo sustentan, lo que está desencadenando un proceso de desarrollo regional (Conafor, 20II).

La silvicultura comunitaria o manejo forestal comunitario (MFC), como es conocido en México, ha sido una apuesta por un modelo de trabajo de autogestión, en la que los propietarios sociales de los bosques han encontrado una forma de aprovechamiento de los recursos forestales, tanto maderables como no maderables, bajo el principio de conservar sus bosques y mantener un aprovechamiento de los suelos que sea sustentable (Ceballos, 20I0).

En el MFC se destacan procesos de apropiación por parte de las comunidades, quienes están aplicado sus usos y costumbres, sus conocimientos locales, así como sus propias formas de organización para desarrollar los trabajos que implica la silvicultura comunitaria, así como la incorporación de valores en el uso de los recursos naturales, para así aprovecharlos de forma integral y generar, además, beneficios económicos (Gasca, 2014).

De acuerdo con el objetivo de este estudio de reconocer el impacto espacial que produce el manejo forestal comunitario, es necesario abordar las teorías del territorio y sus interacciones, las cuales resultan tan complejas como las mismas relaciones sociales; de acuerdo con lo anterior, se puede inferir que no se debe estudiar el territorio separado de las actividades colectivas intrínsecas del ser humano que allí se practican, debido a la íntima relación que entre estos se establece (Garrocho, 20I6). La complejidad que adquiere el estudio del territorio nace a partir de las relaciones sociales que el hombre establece con su entorno, vinculado a todos los ámbitos del desarrollo, construyendo, reconstruyendo o reinventando este (Mota Díaz y Sandoval Forero, 2006).

Gasca (2009) menciona que el territorio es una concepción que está directamente relacionada con la propiedad, la identidad y el gobierno, con la organización de espacios, derechos y límites; de hecho, existe una importante relación con el concepto de región, el cual proviene de la expresión latina regio, "que indicaba la dirección en línea 
recta trazada en el cielo por los augures para delimitar sus partes” (Gasca, 2009, p. 34). De ahí proviene la raíz reg, de la que se construyen las palabras reino, regla, regente, etcétera. El mismo concepto de región se relaciona etimológicamente con el verbo regere, que significa 'dirigir, mandar, guiar, poner límites'. En ese sentido, el territorio es una región política bien definida, un espacio físico de la superficie terrestre delimitado y ordenado bajo ciertos principios y normas de gobierno (Llanos, 2010).

Desde una perspectiva sociológica, el territorio refleja la multidimensionalidad de la vida de los miembros de una colectividad y de las sociedades en general (Rivera, 2006). Los hombres viven al mismo tiempo el proceso y el producto territorial, mediante un sistema de relaciones preexistentes y productivas, de las cuales no siempre son conscientes. Dichas relaciones se basan en estructuras de poder entre los actores y su territorio (Raffestin, 20II, p. II2).

Por su parte, la nueva geografía económica (NGE) ha fortalecido la teoría económica del territorio, la cual ha producido muchos estudios en la actualidad que se cimientan sobre variables de crecimiento y desarrollo, a partir de una teoría que toma en cuenta las características territoriales (Fujita y Krugman, 2004). No obstante, cabe aclarar que la NGE basa sus postulados en supuestos de determinada racionalidad económica, como el libre mercado, la competencia, los derechos de propiedad, etcétera. En ese sentido, este tipo de racionalidad no es compatible al cien por ciento con el MFC, en el que se posee una racionalidad ambiental y social mucho más amplia. Debido a ello, es necesario recurrir a otras perspectivas más amplias del concepto de territorio e incluso de desarrollo; es por ello que se explicaba esta parte sociológica del territorio, donde se ven reflejadas relaciones sociales de poder y dependencia, entre otras.

Este punto es muy importante desde la noción de la autogestión y del MFC, ya que, como mencionábamos anteriormente, la definición del MFC se encuentra en su forma de organización, la relación con los medios de producción y con el territorio.

$\mathrm{Al}$ respecto, algunos autores han mencionado que los dos bastiones del MFC son la autodeterminación y la comunalidad (Arias, 20I8; Fuente, 20I2): mientras que en los modelos de desarrollo local tradicional se busca imponer objetivos, proyectos, metas, y utilizar a sus pobladores solo como mano de obra barata, el MFC se ha construido sobre un modelo de desarrollo sustentable, con sus propios objetivos y metas, y se ha buscado la autogestión de estos, con base en sus propias reglas.

Por otra parte, en cuanto al impacto, una forma de identificar si son posibles las prácticas sustentables a partir del MFC es analizando el territorio. En sintonía con lo anterior, la Conafor lanzó en 2006 la primera convocatoria para realizar los Estudios Regionales Forestales de las Diferentes Unidades de Manejo Forestal (Umafor) en el país, y brindaron una guía para la elaboración de estos. En dicha guía se solicita como parte de los resultados la elaboración de una matriz de cambios de cobertura, considerando las cartas de uso del suelo y vegetación Serie II (2000) y III (2003). No obstante, para la Sierra Norte de Puebla se obtuvo su matriz a partir de los cambios de cobertura con la Serie I (1986) y la Serie II (2000), únicamente para bosques y matorrales, lo cual arrojó pérdidas de bosque por un total de $240 \mathrm{~km}^{2}$ y $139 \mathrm{~km}^{2}$ de degradación (Conafor, 2009).

\section{Metodología}

La metodología utilizada en este artículo es de tipo mixta, pues está basada tanto en análisis cuantitativo y como cualitativo. Los materiales utilizados para la primera etapa del estudio constataron de información de segunda mano con carácter oficial, tales como los planes de manejo, los estudios regionales forestales, la revisión de las bases de datos del Padrón e Historial de Núcleos Agrarios del Registro Agrario Nacional (Phina), del que se obtuvo información de las carpetas básicas de los ejidos, tomando como referencia aquellos que poseen 
tierra de uso común. En los indicadores económicos se utilizó el Sistema Nacional de Información Ambiental y Recursos Naturales de la Secretaria de Medio Ambiente y Recursos Naturales (Semarnat, 2018) y el Banco de Indicadores: Agricultura, ganadería, y pesca del Instituto Nacional de Estadística y Geografía (Inegi, 2018), tomando como referencia el volumen y valor de la producción forestal maderable y no maderable. Por último, se obtuvo información de los apoyos y subsidios forestales otorgados a las comunidades de la región para contrastar el papel de la inversión pública versus el desarrollo local y regional.

Para la realización de las tablas de cambios de cobertura, se utilizaron como base las Series III y VI de Inegi, mediante las capas de información de Uso del Suelo y Vegetación Proyección Área Equivalente de Albers ITRF 1992, con las cuales se realizó un cruzamiento de la información de ambas capas, mediante el programa ArcGis, versión i0.5. La clasificación utilizada fue la proporcionada por Inegi, la cual está siendo trabajada dentro del proyecto internacional "Las Cuentas Experimentales de los Ecosistemas", y que a su vez considera la clasificación de la Comisión Nacional Forestal (Conafor), con el propósito de tener una mayor representatividad del aprovechamiento de los bosques (véase la tabla I).

TABLA 1.

UNIDADES DE ANÁlISIS MEDIANTE LOS TIPOS DE USO DEL SUELO

\begin{tabular}{|c|c|c|}
\hline N. $^{\circ}$ & Clave & Descripción \\
\hline 1 & AGR_AN & Agricultura anual \\
\hline 2 & AGR_PER & Agricultura permanente \\
\hline 3 & $\mathrm{AH}$ & Asentamientos humanos \\
\hline 4 & $\mathrm{BCO} / \mathrm{P}$ & Bosque de coniferas primario \\
\hline 5 & $\mathrm{BCO} / \mathrm{S}$ & Bosque de coniferas secundario \\
\hline 6 & $\mathrm{BE} / \mathrm{P}$ & Bosque de encinos primario \\
\hline 7 & $\mathrm{BE} / \mathrm{S}$ & Bosque de encinos secundario \\
\hline 8 & $\mathrm{BM} / \mathrm{P}$ & Bosque mesófilo de montaña primario \\
\hline 9 & $\mathrm{BM} / \mathrm{S}$ & Bosque mesófilo de montaña \\
secundario
\end{tabular}

Fuente: elaboración propia con base en Ocampo (2014) y la Organización de las Naciones Unidas (ONU, 2012).

La información de los cambios de cobertura del suelo nos brindó la información necesaria para conocer la cobertura del suelo en dos momentos diferentes, al inicio y al final del periodo de referencia, así como los incrementos y las reducciones de esas superficies, según los diferentes tipos de cobertura (ONU, 20I2, p. 155). Es importante señalar que la información de cambios de cobertura pueden presentar también algún porcentaje de error, que podrían sortearse con el mejoramiento de las imágenes tomadas por la tecnología disponible o de las herramientas de cálculo y fotointerpretación. Al respecto, Camacho et al. (2015) recomiendan la metodología 
de Mas et al. (2003) para la identificación de errores y la forma de corregir estos, mediante la evaluación de la confiabilidad temática; no obstante, este método no se utilizó en el estudio, debido a que el porcentaje de error fue significativamente menor.

En cuanto a las unidades y categorías de análisis, encontramos, en un primer momento, que a partir de la clasificación utilizada (la de Conafor) contamos con is tipos de áreas, en las que se destacan los bosques de coníferas, bosques mesófilos de montaña y bosques de encino, además de otros usos de suelo, tales como el agrícola y el urbano (asentamientos humanos); por el contrario, si hubiéramos utilizado la clasificación de Inegi, tendríamos que haber utilizado 76 tipos de áreas, por lo cual se decidió tomar la clasificación de Conafor para destacar más los aspectos productivos forestales que su biodiversidad (véase el anexo i).

Por último, se realizaron recorridos en campo para contrastar la información, así como la realización de entrevistas estructuradas y dirigidas a técnicos forestales y representantes de los ejidos. Producto de estas entrevistas, se obtuvo información sobre el desarrollo forestal, la defensa y apropiación de los recursos forestales, la vida en comunidad y las relaciones que ellos establecen con otros actores sociales e institucionales para el aprovechamiento sustentable de los bosques; esto se presenta como un ente articulador para el desarrollo del territorio, la economía y la población, con el propósito de conocer cuál ha sido la incidencia de la actividad forestal en los ejidos de esa región. Lo anterior se incluyó como parte de la metodología, considerando lo que menciona Arias (2018), citando a Quijano (1992), sobre el decolonialismo del conocimiento y de las ciencias posnormales (Funtowicz y Ravetz, 1993), en los que la expresión de los actores o sujetos de investigación son imprescindibles para tener un enfoque más amplio y real del problema.

\section{Área de estudio}

Las áreas boscosas en el territorio mexicano están habitadas en su mayoría por ejidos y comunidades, $55 \%$, cifra que es obtenida mediante el cálculo con información del Registro Agrario Nacional (2012) y la Serie III, los cuales obtienen el porcentaje de bosques y selvas en propiedad social en el país. Lo anterior forma parte de las interacciones sociales y ecosistémicas que se establecen dentro de dichos territorios y, en consecuencia, requieren de formas de aprovechamiento armónicas para el bienestar social y el manejo forestal sustentable.

El área de estudio se ubica en la Región Sierra Norte de Puebla, la cual se localiza al norte del estado y colinda con los estados de Hidalgo, hacia el oeste, y con el de Veracruz, hacia el sur. Esta región forma parte de las provincias fisiográficas Eje Neovolcánico y la Sierra Madre Oriental; se integra por cuatro municipios: Aquixtla, Chignahuapan, Ixtacamaxtitlán y Zacatlán, que abarcan un total de $1976.82 \mathrm{~km}^{2}$ (Inegi, 2017b). Así mismo, esta sierra forma parte de la Unidad de Manejo Forestal (Umafor) 2108, "Zacatlán”, conformada por iz ejidos en total, de los cuales los 4 municipios seleccionados abarcan el 72.3\% de esta. En ese sentido, la Sierra Norte del Estado de Puebla es la región forestal más importante del estado.

La Sierra Norte de Puebla pertenece casi en su totalidad a la Región Hidrológica “RH27”, de la cuenca del río Tecolulta, en las subcuencas de los ríos Necaxa, Ajajalpan Apulco y Blanco. Solamente una zona pequeña del oeste se identifica como parte de la Región Hidrológica “RH26” de la cuenca del río Panuco.

En el territorio predominan los climas templados, tales como el templado subhúmedo con lluvias en verano de humedad media $C\left(w_{1}\right)(w)$, templado subhúmedo con lluvias en verano $C\left(w_{2}\right)$ y semifrío subhúmedo con lluvias en verano $C(E)\left(w_{2}\right)$. La zona que se encuentra bajo la influencia de este clima comprende los municipios de Chignahuapan, Aquixtla e Ixtacamaxtitlán, mientras que para Zacatlán el clima predominante es el templado húmedo con lluvias abundantes en verano $\mathrm{C}(\mathrm{m})$. La temperatura media anual varía entre ı2o y ı⿻oC; la precipitación total anual tiene un rango de 600 a $1000 \mathrm{~mm}$, y el porcentaje de lluvia invernal es menor a 5 . 


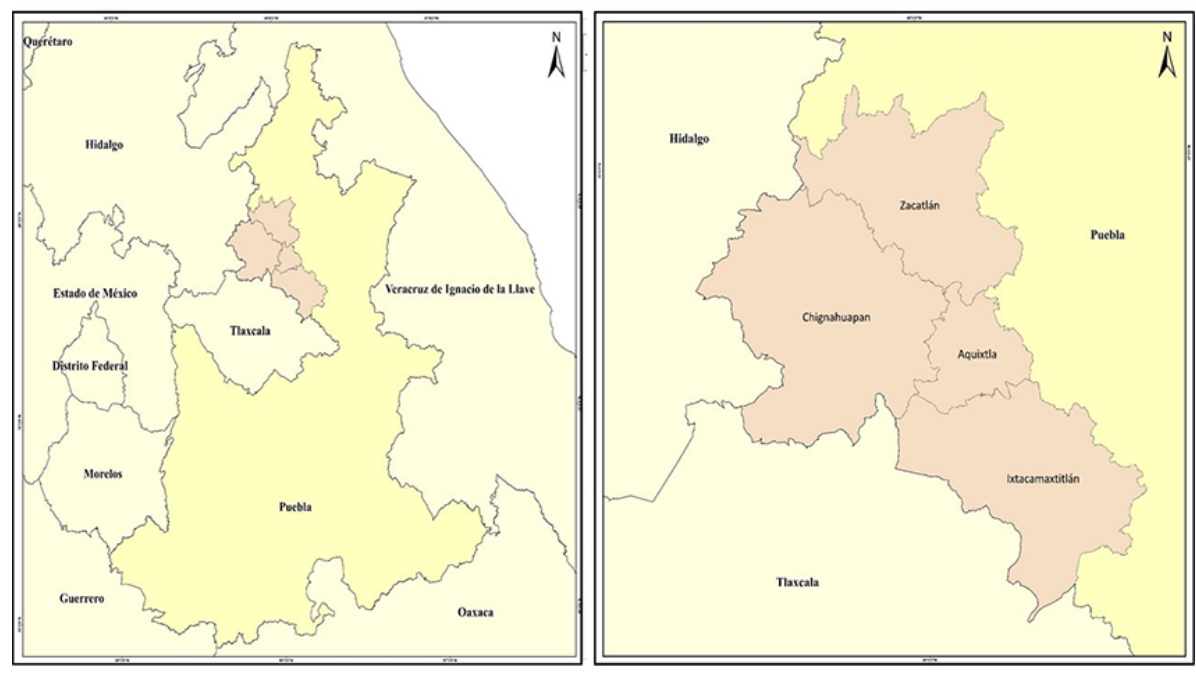

FIGURA 1.

Ubicación de la Sierra Norte de Puebla

Fuente: elaboración propia con base en el Marco Geostadístico del Inegi (2010).

En cuanto a la conformación del territorio, que consta de $1976.82 \mathrm{~km}^{2}$, en él existen 85 núcleos agrarios que ocupan una superficie del $32 \%$; sin embargo, la superficie de uso común dentro del núcleo agrario representa el $50.7 \%$ (véase la tabla 2). La población contabilizada en la región para el 20ı0, por el Censo de Población y Vivienda, fue de 167379 personas, de las cuales 80785 son hombres y 86594 son mujeres. Curiosamente, el municipio con mayor población no es el de mayor territorio, sino Zacatlán con 76296 habitantes. El Índice de Marginación, de acuerdo con Conapo (2010), varía entre medio, para los municipios de Chignahuapan y Zacatlán, y alto para Aquixtla e Ixtacamaxtitlán; lo anterior se ve reflejado, por ejemplo, en el grado de escolaridad de los municipios con mayor marginalidad, en donde el $40 \%$ de su población no completó la educación primaria, y un aproximado de $20 \%$ de población no sabe leer ni escribir. Los municipios con grado de marginalidad medio tienen una tasa de analfabetismo del I $3 \%$, y el porcentaje de población que no completó la primaria es del $30 \%$.

TABLA 2.

Superficie del territorio, núcleos agrarios y de uso común en la Sierra Norte de Puebla (Km²)

\begin{tabular}{|l|c|c|c|c|}
\hline \multicolumn{1}{|c|}{ Municipios } & Territorio & Núcleos agrarios & Superficie del núcleo & Superficie de uso común \\
\hline Aquixtla & 166,81 & 7 & 25,38 & 20,67 \\
\hline Chignahuapán & $\mathbf{7 5 9 , 6 8}$ & 45 & 430,09 & 219,07 \\
\hline Ixtacamaxtitlán & 561,12 & 20 & 157,46 & 70,95 \\
\hline Zacatlán & 489,2 & 13 & 37,18 & 18,81 \\
\hline \multicolumn{1}{|c|}{ Total } & $\mathbf{1 . 9 7 6 , 8 2}$ & $\mathbf{8 5}$ & $\mathbf{6 5 0 , 1 2}$ & $\mathbf{3 2 9 , 5}$ \\
\hline
\end{tabular}

Fuente: elaboración propia con base en Ran (2018) e Inegi (2005; 2017a).

En cuanto a las características de los asentamientos, la concentración de las localidades no rebasa los 5 mil habitantes, especialmente en Aquixtla e Ixtacamaxtitlán, eso significa que la mayoría de las personas vive en un contexto rural. Las cabeceras municipales de Chignahuapan y Zacatlán se pueden considerar como pequeñas ciudades. Las características de las viviendas cuentan con algún tipo de precariedad, tales como piso de tierra (I4\%), falta de agua entubada (15\%), falta de electricidad (2.3\%). Finalmente, la proporción de la población que vive con 2 salarios mínimos o menos es el $71 \%$ (Conapo, 2010) 


\section{Análisis de los resultados}

\section{Producción}

La Sierra Norte de Puebla es la región -en cuanto a producción forestal maderable- más importante del estado de Puebla. Tan solo el volumen de la producción maderable de la región, para el año 2015, alcanzó la cifra de 179 $962 \mathrm{~m}^{3}$ rollo (véase la figura 2), la más alta en el periodo de estudio, lo cual representa el 6ı.7\% de la producción total de madera del estado (Inegi, 20r7b).

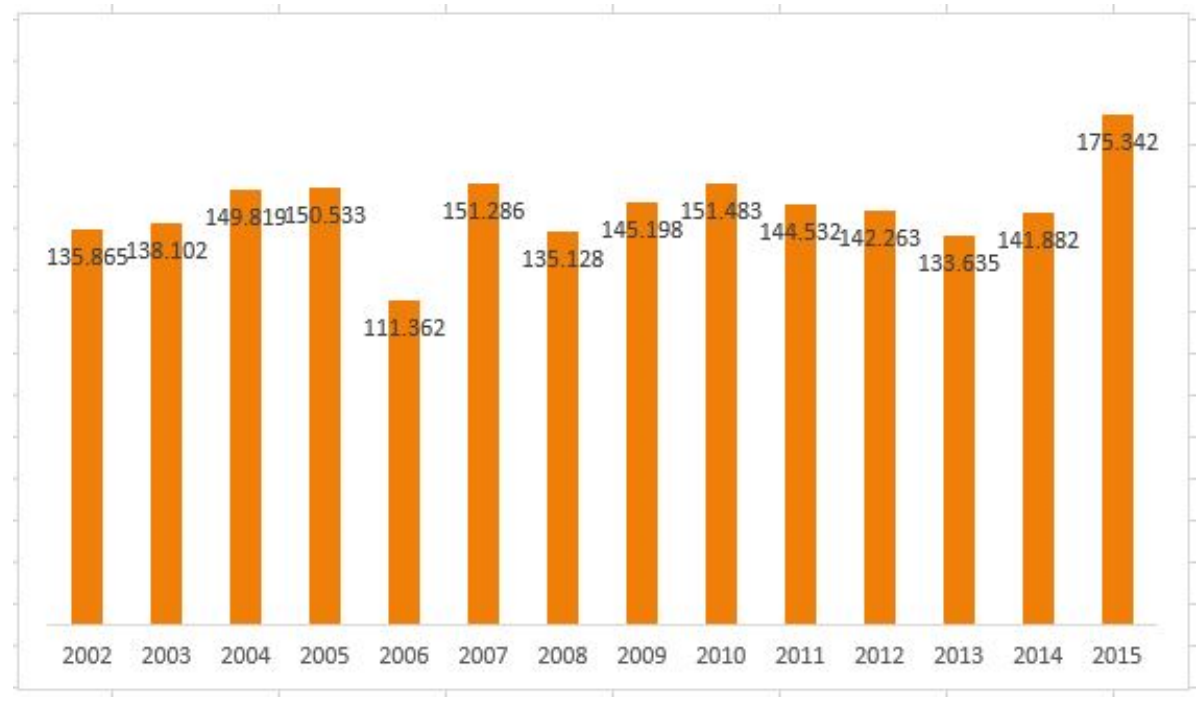

FIGURA 2.

Volumen de la producción forestal maderable en la Sierra Norte de Puebla, 2002-2015 (metros cúbicos rollo)

Fuente: elaboración propia con base en Semarnat (2018).

En cuanto al precio promedio de la madera, obtenido mediante el valor de la producción dividido entre el volumen de producción por año, se observa una fluctuación constante, alcanzando como valor máximo I235 pesos por metro cúbico de rollo en el 2003 y como precio mínimo 97ı pesos en el año 2009 (véase la figura 3). Una forma en la que se podría aumentar el valor de la producción es la del mejoramiento o transformación de los productos. Sin embargo, la clasificación de los productores no ha cambiado en el periodo de estudio, no obstante, algunos ejidos sí están trabajando en buscar la forma de obtener mejores rendimientos de su producción, a través de la actualización de sus procesos productivos y con la adquisición de tecnologías y búsqueda de nuevos mercados. 


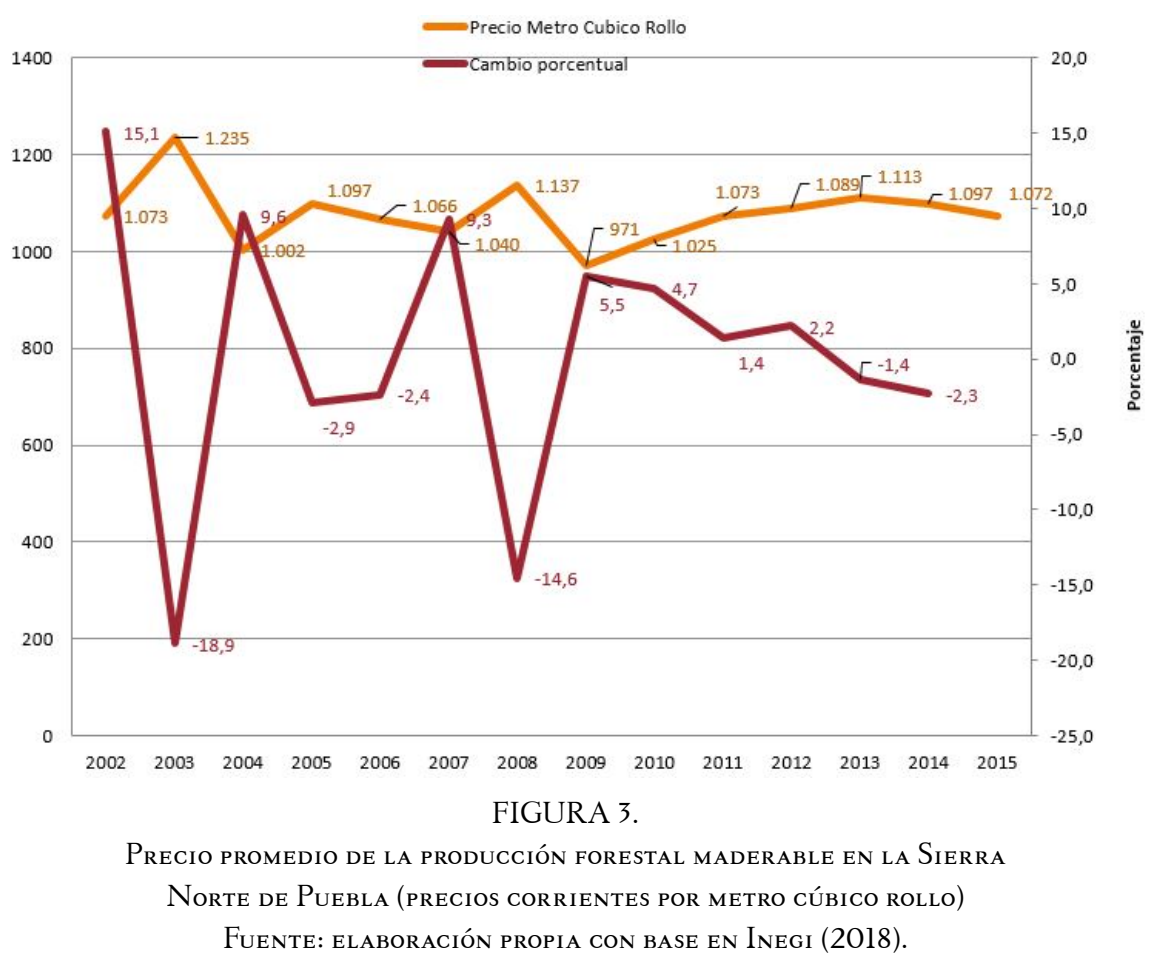

En cuanto a la forma de producción forestal, la Sierra Norte de Puebla cuenta con diferentes sistemas de manejo, entre los que destacan el método de desarrollo silvícola (MDS), con 77.73\% de la superficie, y el método mexicano de ordenación de montes o método mexicano de ordenación de bosques irregulares (MMOBI), con 13.81\% de la superficie; el resto, se da a partir de otros métodos, tales como el sistema de conservación y desarrollo silvícola (Sicodesi) o el método de melección o mezclados (véase la figura 4).

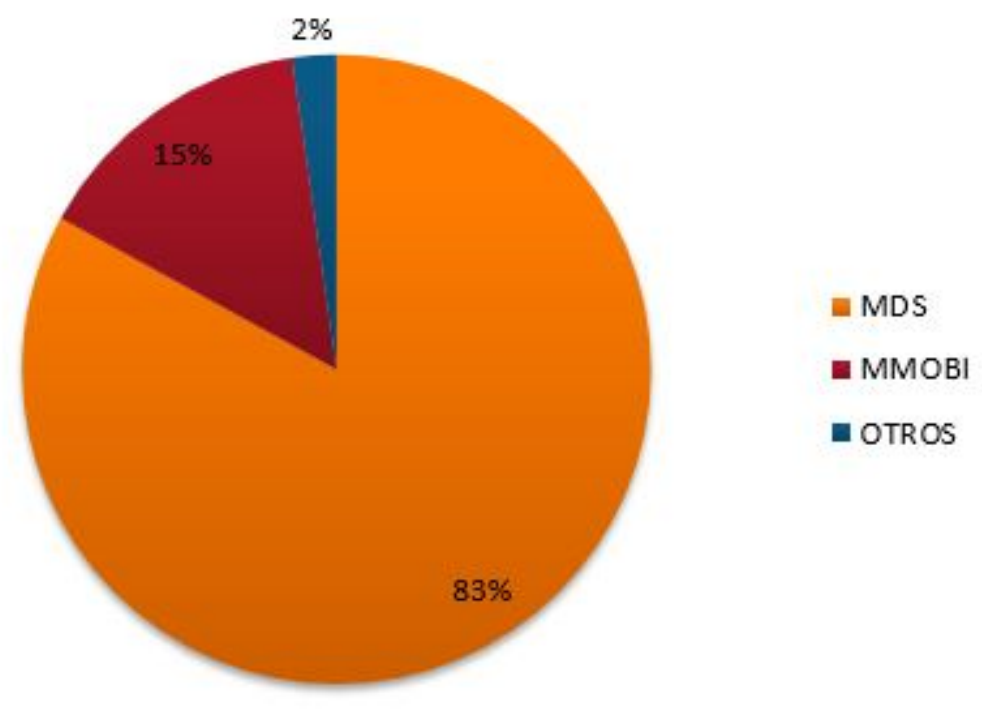

FIGURA 4.

Métodos de aprovechamiento forestal en la Sierra Norte de Puebla Fuente: Conafor (2009).

Grosso modo, el método de desarrollo silvícola consiste en estimar el volumen de cosecha en función de una serie de reglas silvícolas, asumiendo un turno y recomendaciones técnicas para la aplicación de tratamientos. El 
MMOBI estima el volumen de cosecha de acuerdo al crecimiento temporal de la población forestal y asume que su recuperación sigue un patrón definido, que se estima mediante una tasa de crecimiento basada en una ecuación de interés compuesto. Los métodos mezclados son muy variados y no siguen ningún patrón sistemático, aunque en la mayoría de los casos resultan ser una combinación entre el MMOBI y el MDS.

Otro factor importante para conocer si se está realizando un manejo forestal sustentable es comparar el nivel de producción forestal con el número de autorizaciones, las cuales se establecen, en primera instancia, en los planes de manejo autorizados por la Semarnat. Se debe tomar en cuenta que puede haber modificaciones a dichas autorizaciones para aumentar las cortas (consideradas como intervenciones silviculturales que pueden aplicarse total o parcialmente) o disminuirlas por eventos no previstos como plagas o incendios. Los planes de manejo, como comentábamos, contribuyen a un manejo forestal sustentable y a la conservación de los bosques. En cuanto al volumen de aprovechamiento maderable con autorizaciones, la Sierra Norte ha mantenido el límite de este como se puede observar en la tabla 3, salvo en el año 2005, cuando hubo un aprovechamiento de $\mathrm{I}_{4} 676 \mathrm{~m}^{3}$ rollo arriba del límite permitido. De acuerdo con la información obtenida de los planes de manejo, algunos planes se encuentran en el tercer ciclo de corta; un ciclo de corta está integrado por diez años y, de acuerdo a las condiciones del bosque, se pueden realizar cortas anuales, bianuales. Los silvicultores suelen utilizar el método de matarraza, árboles padres o el método de bosques irregulares, esto se da en función de las condiciones naturales en que se encuentre el bosque, lo cual debe quedar establecido en el programa de manejo forestal.

TABLA 3.

Aprovechamiento forestal maderable autorizado y cosechado

en la Sierra Norte de Puebla, 2002-2015 (metros cúbicos rollo)

\begin{tabular}{|c|c|c|c|c|c|c|c|c|c|c|c|c|c|c|}
\hline & 2002 & 2003 & 2004 & 2005 & 2006 & 2007 & 2008 & 2009 & 2010 & 2011 & 2012 & 2013 & 2014 & 2015 \\
\hline $\begin{array}{l}\text { Volumen de aprovechamiento forestal maderable } \\
\text { autorizado para el año (metros cúbicos rollo) }\end{array}$ & 431251 & 387555 & 183517 & 135857 & 174198 & 176762 & 180661 & 171914 & 176860 & 177057 & 198144 & 195731 & 188996 & 225314 \\
\hline $\begin{array}{l}\text { Volumen de la producción forestal maderable } \\
\text { (metros cúbicos rollo) }\end{array}$ & 135865 & 138102 & 149819 & 150533 & 111362 & 151286 & 135128 & 145198 & 151483 & 144532 & 142263 & 133635 & 141882 & 175342 \\
\hline Diferencia & 295386 & 249453 & 33698 & -14676 & 62836 & 25476 & 45533 & 26716 & 25377 & 32525 & 55881 & 62096 & 47114 & 49972 \\
\hline
\end{tabular}

Fuente: elaboración propia con base en Semarnat (2018).

\section{Caracterización de los productores}

La mayor parte de los predios con autorización de aprovechamiento forestal está en manos de los ejidos, los cuales incrementaron 55.4\%: el número de autorizaciones pasó de 531 a 825, del año 2000 al 2015; de igual forma, la superficie autorizada creció en II $\%$, pasando de $269 \mathrm{~km}^{2}$ a $57 \mathrm{I} \mathrm{km}{ }^{2}$. Así mismo, con base en información del Phina y de la Conafor, en la región existe la siguiente tipología de ejidos forestales que realizaron aprovechamiento forestal con programas previamente autorizado por la Semarnat: del tipo I, hay 22 núcleos agrarios; del tipo II, fueron 49; del tipo III, fueron 5, y del tipo IV, fueron 2. Lo anterior da un total de 78 ejidos y los años en los que obtuvieron sus permisos van del 200 I al 2008. La tipología comprende desde los que venden en pie hasta los que realizan algún proceso de transformación industrial de la madera.

Esta tipología es la que ha servido como base para la entrega de apoyos de la Conafor: para el periodo de 2011 a 20I5, se otorgaron 814 apoyos a la región, de los cuales corresponden el $39 \%$ para silvicultura y caminos forestales; el 13\% se distribuyó para restauración forestal y reconversión productiva; el $9 \%$ para estudios y proyectos, en los que entran la elaboración de programas de manejo forestal, manifestaciones de impacto ambiental, estudios y documentos técnicos, y planes de negocios; el 22\% para el desarrollo de capacidades, que consiste en evaluaciones rurales participativas, seminarios de comunidad a comunidad, ordenamientos territoriales 
comunitarios, conformación de comités de vigilancia, capacitación de promotores forestales comunitarios, talleres y cursos, y planes prediales de mediano plazo (véase la figura 5).

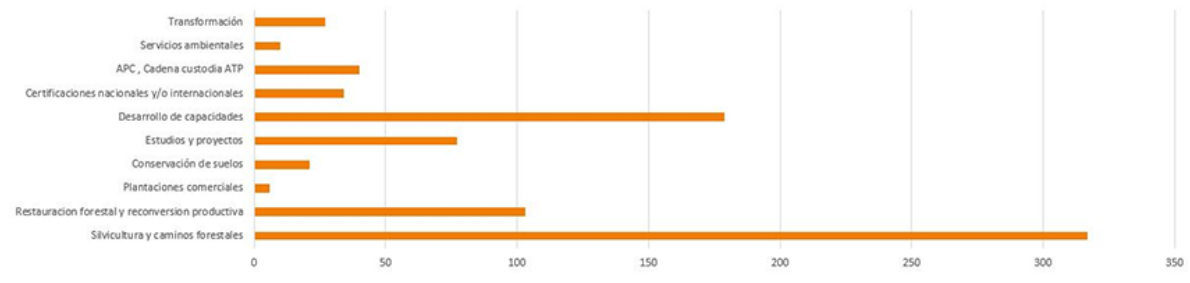

FIGURA 5.

Apoyos forestales otorgados a la Sierra Norte de Puebla, 2011-2015

FuENTE: ELABORACIÓN PROPIA CON BASE EN RAN (2018).

Los ejidos que han recibido un mayor número de apoyos de desarrollo de capacidades son en general aquellos que se encuentran certificados, que poseen industria, que generan servicios ambientales: en Aquixtla, el ejido de Terreros recibió 7 apoyos; en el municipio de Chignahuapan, los ejidos de Llano Grande ro apoyos y Peñuelas Pueblo Nuevo 8 apoyos; en Ixtacamaxtitlán, el ejido Calpaneria 9 apoyos; finalmente, en el municipio de Zacatlán, el ejido Tulimán recibió 8 apoyos y Nanacamila 6 apoyos.

Los apoyos en acreditación para procesos de certificación, cadena de custodia, auditoría técnica preventiva, ferias y actas constitutivas fueron del $5 \%$. De este porcentaje, solo el $0.4 \%$ pertenece al concepto de apoyo para la certificación de la cadena de custodia; los ejidos beneficiados fueron Llano Grande, Peñuela Pueblo Nuevo y Piedra Ancha Segunda Sección, pertenecientes al municipio de Chignahuapan y el ejido Nanacamila del municipio de Zacatlán.

En lo referente a servicios ambientales, se recibieron ıо apoyos (г\%), 9 pertenecen a ejidos del municipio de Chignahuapan y uno al ejido de Nanacamila, Zacatlán. Para la adquisición de equipo de transformación, únicamente se recibió el $3 \%$, lo que indica que en la región los ejidos en general no cuentan con equipo de trasformación que les ayude a darle valor agregado a sus productos.

La certificación forestal, con base en lo que establece la Norma Mexicana NMX-AA-I43-SCFI-2008 para la certificación del manejo sustentable de los bosques, es un requisito voluntario, hasta el momento, que deben cumplir los que aprovechan bosques, en términos de dar mayor certeza y reconocimiento a la legalidad del aprovechamiento forestal. El municipio que cuenta con un mayor número de ejidos con certificación nacional es Chignahuapan, con ir ejidos certificados y 3 en proceso de dictamen. Zacatlán, con un ejido certificado (Nanacamila); Ixtacamaxtitlán, con un ejido en proceso (Minillas), y Aquixtla, con una fracción privada en proceso de certificación (Chichicaxtla), esto en 2015. Asimismo, el municipio de Chignahuapan es el que cuenta con mayores certificaciones internacionales, con un total de 6, y Zacatlán únicamente cuenta con una en el ejido de Nanacamila, estos emitidos por Rainforest Alliance; se trata de ejidos que tienen ambas certificaciones, la nacional y la internacional. No obstante, las certificaciones, aun cuando dan certidumbre sobre la evaluación del manejo forestal sustentable, solo cubren algunos predios.

\section{Cambios de cobertura de uso del suelo}

Los cambios de cobertura de uso del suelo se pueden analizar de distintas formas, una de ellas, es elaborar un cuadro en el que se observen los datos de entrada y salida, es decir, el periodo de inicio y el periodo final (véase la tabla 4). A través de ella, podemos observar que los mayores cambios en términos de espacio se dieron en agricultura anual y pastizal, con pérdidas y ganancias en bosques de coníferas primario y secundario. Otros usos del suelo, 
como asentamientos humanos, se incrementaron en un $133 \%$, sin embargo, en términos absolutos, el incremento solo es de $5.46 \mathrm{~km}^{2}$.

TABLA 4.

Cambios en el uso del suelo neto en la Sierra Norte de Puebla (kilómetros cuadrados)

\begin{tabular}{|l|r|r|r|}
\hline \multicolumn{1}{|c|}{ USO DE SUELO } & USV III (2002) & \multicolumn{1}{c|}{$\begin{array}{c}\text { USV VI } \\
\text { (2013) }\end{array}$} & \multicolumn{1}{c|}{ USV III-USV VI } \\
\hline Agricultura anual & $1,034,06$ & 986,16 & $-47,90$ \\
\hline Agricultura permanente & 9,31 & 6,82 & $-2,48$ \\
\hline Asentamientos humanos & 4,10 & 9,56 & 5,46 \\
\hline Bosque de coniferas primario & 608,22 & 643,19 & 34,97 \\
\hline Bosque de coniferas secundario & 138,29 & 170,21 & 31,92 \\
\hline Bosque de encinos primario & 32,09 & 32,95 & 0,87 \\
\hline Bosque de encinos secundario & 9,90 & 5,41 & $-4,50$ \\
\hline Bosque mesófilo de montaña primario & 6,15 & 7,16 & 1,01 \\
\hline Bosque mesófilo de montaña secundario & 5,91 & 5,42 & $-0,49$ \\
\hline Cuerpos de agua & 1,68 & 2,24 & 0,56 \\
\hline Matorral xerofilo no leñoso primario & 40,24 & 40,31 & 0,07 \\
\hline Otras tierras & 0 & 1,48 & 1,48 \\
\hline Pastizal & 86,88 & 65,93 & $-20,95$ \\
\hline \multicolumn{1}{|c|}{ Total } & $1.976,82$ & $1.976,82$ & $-1.976,82$ \\
\hline
\end{tabular}

Fuente: elaboración propia con base en Inegi (2005; 2017a).

\section{Discusión}

La presente discusión analiza la información consignada en los párrafos anteriores, además de evaluar las observaciones y los resultados de las entrevistas dirigidas, buscando cumplir con lo que se mencionaba en el apartado de la metodología sobre la importancia de tener en cuenta la opinión de los sujetos de investigación.

En ese sentido, el impacto del MFC forma parte del proceso y el producto territorial que se lleva a cabo. Aunque las cifras de producción o explotación forestal maderable han aumentado, esto ha sido a través de planes de manejo forestal autorizados y, de acuerdo con la información de la tabla 4, puede considerarse que existe en la región un desarrollo forestal sustentable.

En el caso de las cuestiones sociales y económicas, existen importantes contrastes, tales como fuertes rezagos en cuestión de ingresos y en el nivel de educación, incluso dentro de la misma región de la Sierra Norte de Puebla, donde Chignahuapan y Zacatlán son los municipios con mayores ingresos, además de ser los que cuentan con el mayor número de apoyos, certificaciones, autorizaciones, niveles de producción y valor de la producción.

Dichas diferencias dentro de una misma región pueden ser originadas por diversas causas, desde la disponibilidad de caminos, las condiciones naturales, el nivel de organización, entre otras. Sin embargo, estos municipios pueden ejercer determinada influencia en derredor de los demás municipios y funcionar como centros gravitacionales para los otros ejidos.

De acuerdo con las entrevistas estructuradas, Los técnicos forestales comentaron que una de las preocupaciones (de los ejidatarios) es el involucramiento de los jóvenes en los procesos de desarrollo territorial y en el manejo forestal comunitario; para cumplir con lo anterior, hace falta que los jóvenes se conviertan en un agente intencional, 
entendido este en términos de las actividades que pueden ser capaces de desarrollar para generar cambios, teniendo en cuenta que ellos son la siguiente generación, a los que se les pasará la estafeta. Sin embargo, y como se observa, los jóvenes ya no quieren trabajar la tierra y abandonan por completo el campo. En ese sentido, el manejo forestal comunitario debe convertirse en un factor que ayude a disminuir la migración de los jóvenes.

Durante los recorridos realizados, en compañía de los técnicos forestales, no hay duda de que el manejo forestal comunitario está ejerciendo una influencia importante en el desarrollo territorial de la región, pero esto podría mejorar si se generan alternativas para un desarrollo más integral, de tal forma que se convierta en un eje articulador de procesos de desarrollo territorial. Las prácticas sociales de trabajo organizado están vivas y se deben potenciar, allí lo económico no se debe constituir como la visión dominante, sino como un complemento de las demás dimensiones del desarrollo; es decir, es imperativo posibilitar mejores ingresos económicos para la comunidad y desarrollar mecanismos de reinversión para contribuir al desarrollo social y para mantener las formas de convivencia solidarias - que ya existen en torno a la práctica silvícola-, en mayor equilibrio con el sistema. Han empezado a aflorar una serie de propuestas, pero depende del poder, en los términos que lo expresa Raffestin (20II).

El poder deberá ser entendido y organizado como el medio para lograr que se hagan cosas - está directamente envuelto en la acción humana-, con el cual el actor territorializa el espacio. Es decir, los cambios deberán partir del propio convencimiento de los actores: sin interés y convicción, lo que prima es la desconfianza y difícilmente se podrá dar cauce a nuevas formas de organización que den pie a otros modos de desarrollo del territorio (Raffestin, 20II, p. I02).

La edad avanzada de los ejidatarios representa una limitante para producir cambios que mejoren los procesos de organización comunitaria y productiva; en muchos ejidos, los ejidatarios tienen entre 50 y 60 años en promedio. Si se encuentra algún joven ejidatario es porque ya falleció la persona que era titular, en realidad, son muy pocos los jóvenes que se han integrado a la comunidad como ejidatarios. En este sentido, hace falta apertura y formación de la población joven para que incursionen en el manejo de los bosques y, de esa forma, se vaya dando paso al relevo generacional; podría decirse que el exceso de regulación y tramitología desestimula el aprovechamiento forestal. No existe el involucramiento de los actores locales en la definición y generación de las políticas públicas forestales, por este motivo, hace falta un mayor empoderamiento por parte de los ejidatarios, es decir, una mayor apertura a la participación para tomar las riendas de su propio desarrollo.

En el ámbito exterior a los ejidos con bosques existe una industria poco articulada, que opera con procesos de baja tecnología, productora principalmente de tarimas. Esta situación habla del potencial forestal que tiene la Sierra Norte de Puebla, el cual no está siendo aprovechado de forma adecuada, por lo que su contribución al desarrollo social también es poco representativa, dado la categoría de alta marginación que presentan la mayoría de los ejidos incorporados en este análisis. En este caso, el progreso no solo debe ser el de las ideas, sino que debe convertirse en el progreso de las formas de producción y de trabajo (Salinas et al., 2017) Este progreso debe ser aplicado en el ámbito del desarrollo local para potenciar las capacidades humanas que existen en la región y hacer frente a las oportunidades que están latentes allí; en algunos casos, se ha logrado que los jóvenes desistan de la idea de migrar, por el hecho de que han visto que la actividad forestal genera empleos.

El mercado donde se comercializa la madera en rollo se encuentra principalmente en el estado de México, Tlaxcala e Hidalgo. No se ha trabajado de forma extendida en los ejidos la idea de la empresa forestal comunitaria, pues los despachos se han orientado a la asistencia técnica y la asesoría de los programas de manejo que consiste en el aprovechamiento y cultivo del bosque. Lo que la Conafor paga es lo que se trabaja y si no hay quien financie el despacho no participa. En las evaluaciones rurales participativas, los asesores tratan de que no solo se enfoque a lo técnico, pero al final siempre se aterriza a lo que es su especialidad, el programa de manejo forestal. La idea original era que los ejidos y comunidades se apropiaran del manejo forestal, pero no ha sido posible, porque hay muchos intereses de por medio. Los ejidatarios generalmente están pensando en cuánto les va a tocar -en términos 
monetarios- del aprovechamiento y no en reinvertir parte de los ingresos que obtienen, y esta reacción se da como mecanismo de sobrevivencia.

En el municipio de Zacatlán, los ejidos más cercanos a la cabecera municipal han hecho la parcelación del área de uso común, como es el caso de Jicolapa: este cuentan con los certificados otorgados por la SRA, lo que permite observar ciertas ventajas a los ejidatarios, pues cada uno de ellos se responsabiliza del cuidado de su parcela, pero, por otro lado, se ha producido una mayor desorganización para la comercialización de la madera.

Actualmente, la actividad que soporta económicamente es la forestal: genera jornales durante el año para el mantenimiento de las áreas forestales que establece el programa de manejo y el reparto de utilidades después del corte, y el reparto de utilidades es de entre ro a 20 mil pesos en promedio, dependiendo del número de ejidatarios.

Los despachos se han enfocado en que los ejidatarios se apropien de las actividades que involucran el manejo y cultivo del bosque. Después, la Conafor a través de la silvicultura comunitaria los ha orientado hacia donde quieren ir, esto a través de talleres de planeación participativa, de estudios de ordenamiento territorial y ahora con los programas prediales de mediano plazo.

\section{Conclusiones}

La complejidad de los procesos de desarrollo sustentable de la Sierra Norte de Puebla es mayor de lo que aparenta. Las metas que ha establecido el MFC en la región no se han alcanzado a percibir del todo, dado que existen otros problemas de tipo social y organizativo que aún no se han logrado superar; sin embargo, dada la senda que se ha trazado, se observa un relativo equilibrio con algunas variables como la cobertura forestal, la producción, la conservación de especies, etcétera. Esto se puede traducir literalmente como un optimismo hacia el uso del MFC y sus expectativas en el corto y mediano plazo, no en el largo, dado que también existe mucha incertidumbre respecto a los precios de la madera y la política forestal, la cual se traduce en apoyos. Se debe destacar la desconfianza para emprender otro tipo de proyectos que no sea de MFC, el papel de los jóvenes como jornaleros en las prácticas silvícolas y la edad avanzada promedio de los ejidatarios, además del desconocimiento de procesos de transformación para otorgarle mayor valor a la madera.

Esto finalmente es el reflejo del impacto territorial del MFC que presenta potencialidades para ser fortalecido, en términos de la sustentabilidad que han logrado los ejidos por el manejo del bosque, de la acción colectiva que ha significado la práctica silvícola y que se puede llevar a otras formas de organización que produzcan procesos de desarrollo integral del territorio.

Existe cierto desconocimiento para acceder a otros mercados que otorguen mejores condiciones de compra y precios para la madera: consideran que hace falta asesoría técnica para integrar valor a la madera y para diversificar las actividades productivas. Los compradores de la madera, en ocasiones, no quieren pagar la madera que se llevaron, argumentando que la madera no es de la calidad estipulada para descontarle al productor. Regularmente, llegan los compradores a buscar la madera, sobre todo cuando requieren volúmenes grandes, y los ejidos le venden a los que llaman el mejor postor. El ejido realiza una asamblea para definir los precios a los que se puede vender: a veces no necesariamente le venden al que ofrece el mejor precio, sino el que da más confianza, en sí, todos los compradores dan un anticipo.

Pensar en una mayor integración de los ejidos forestales, en términos de la conformación de redes de valor para propiciar procesos de desarrollo local articuladas, no se visualiza como una posibilidad de mediano plazo. La industria que domina en la región es la de las tarimas, por lo que los aserraderos se han orientado más a producir madera para esta industria, generando con ello un desperdicio de madera de mayor calidad, que bien 
podría ser utilizada para otro tipo de productos de mayor valor; entre otras cosas, los apoyos institucionales tienden a concentrarse para ciertos ejidos.

Pese a las asimetrías que pueda presentar el MFC en la Sierra Norte de Puebla, este modelo ha sido un detonador del desarrollo, reduciendo con ello la migración de los jóvenes, ya que las labores del manejo del bosque generan empleos para realizar los chapeos, podas, aclareos y el derribo de los árboles que fueron previamente marcados para el aprovechamiento forestal.

Por último, la Sierra Norte de Puebla es una región donde el MFC está cada vez más integrado por parte de los ejidos que la practican y se observa un mantenimiento de la cobertura forestal, incluso con incrementos -observados en la tabla i de cambios de cobertura de usos del suelo-, así como en los recorridos realizados. Lo anterior es producto de la aplicación de sus programas de manejo que derivan en buena medida del trabajo cooperativo y el aprovechamiento forestal regulado con base en la NOM-152-Sermanat-2006 y la Ley General de Desarrollo Forestal Sustentable (LGDFS), y con apego a la Ley Agraria, en términos de cumplir con las faenas, las brigadas de vigilancia y, de no cumplirse, con la aplicación de las sanciones para el ejidatario, tales como multas o la posibilidad de no recibir los ingresos que corresponden producto de la venta de la madera.

\section{Referencias}

Arias, A. (2018). From Indigenous Literatures to Native American and Indigenous Theorists: The Makings of a Grassroots Decoloniality. Latin American Research Review, 53(3), 613-626. https://doi.org/10.25222/larr.I8I

Bray, D. y Merino, L. (2005). La experiencia de las comunidades forestales en México, veinticinco años de silvicultura y construcción de empresas forestales comunitarias. Instituto Nacional de Ecología (INE-Sermanat).

Bray, D., Merino, L. y Barry, D. (2007). El manejo comunitario en sentido estricto: las empresas forestales comunitarias de México. En D. Bray, L. Merino y D. Barry (Eds.), Los bosques comunitarios de México, manejo sustentable de paisajes forestales (pp. 21-50). Sermanat, Instituto Nacional de Ecología, Instituto de Geografía de la UNAM, CCMSS y Florida International University.

Camacho, J., Pérez, J., Pineda, N., Cadena, E., Bravo, L. y Marcela, M. (2015). Cambios de cobertura/uso del suelo en una porción de la Zona de Transición Mexicana de Montaña. Madera y Bosques, 2I(I), 93-II2. https://do i.org/10.21829/myb.2015.211435

Ceballos, S. (2010). Retos y perspectivas del manejo forestal comunitario en la Sierra Norte de Oaxaca (tesis para obtener el grado de Doctor en Economía). Facultad de Estudios Superiores Aragón, Posgrado en Economía, UNAM.

Comisión Nacional Forestal [Conafor]. (2009). Inventario Nacional Forestal y de Suelos 2004-2009. Recuperado el I3 de octubre de 2018 de https://www.conafor.gob.mx/biblioteca/Inventario-Nacional-Forestal-y-de-Suelos.pdf

Comisión Nacional Forestal [Conafor]. (20II). Memoria del Encuentro Nacional de Silvicultura Comunitaria, Gerencia de Silvicultura Comunitaria, Ciudad de México.

Consejo Nacional de Población [Conapo]. (2010). Indice de Marginación por entidad federativa y municipio 2010. Recuperado el I2 de enero de 2018 de http://www.conapo.gob.mx/es/CONAPO/Indices_de_Marginacion _2010_por_entidad_federativa_y_municipio

Fuente, M. (2012). La comunalidad como base para la construcción de resiliencia social ante la crisis civilizatoria. Revista Latinoamericana Polis, II(33), 195-218. http://dx.doi.org/10.4067/S0718-65682012000300009

Fuente, M. y Carcaño, E. (2016). La Nueva Ruralidad Comunitaria como estrategia de gobernanza local frente al paradigma neoliberal. En G. Rodríguez, L. Álvarez, D. Tagle y J. Coronado (Coords), Desarrollo desde lo local y Dinámicas Territoriales (pp. 219-239). Fontamara y Universidad de Guanajuato. 
Fujita, M., y Krugman, P. (2004). La nueva geografía económica: pasado, presente y futuro. Investigaciones Regionales, (4), 177-206. https://www.redalyc.org/pdf/289/28900409.pdf

Funtowicz, S. y Ravetz, J. (1993), La ciencia posnormal: Ciencia con la gente.. Icaria - Antrazyt.

García, G. and Antinori, C. (2018). Between Grassroots Collective Action and State Mandates: The Hybridity of Multi-Level Forest Associations in Mexico. Conservation and Society, I6(2), 193-204. https://doi.org/10.4103/ CS.CS_I6_II5

Garrocho, C. (2016). Ciencias sociales espacialmente integradas: la tendencia de Economía, Sociedad y Territorio. Economía, Sociedad y Territorio, XVI(50), I-20. http://www.scielo.org.mx/scielo.php?script=sci_arttext\&pid=SI 405-8421201600010000I

Gasca, J. (2009). Geografía regional: La región, la regionalización y desarrollo regional en México (textos monográficos). IGUNAM, México.

Gasca, J. (20I4). Gobernanza y gestión comunitaria de recursos naturales en la sierra norte de Oaxaca. El Colegio de Sonora.

Instituto Nacional de Estadística y Geografía [Inegi]. (2005). Carta de Uso del Suelo y Vegetación, Serie III. Recuperado el I5 de julio de 2018 https://www.inegi.org.mx/temas/usosuelo/default.html\#Descargas,

Instituto Nacional de Estadística y Geografía [Inegi]. (2010). Marco Geoestadístico Nacional. Aguascalientes, Inegi. Recuperado el 28 de agosto de 2018 de https://www.inegi.org.mx/temas/mg/

Instituto Nacional de Estadística y Geografía [Inegi]. (2014). Diccionario de Datos de Uso del Suelo y Vegetación I:250,00o. Recuperado el 4 de octubre de 2018 de http://wwwz.inegi.org.mx/contenidos/temas/mapas/usosuelo/met adatos/dd_usyv_v3_25ok.pdf

Instituto Nacional de Estadística y Geografía [Inegi]. (2017a). Carta de Uso del Suelo y Vegetación, Serie VI. Recuperado el I5 de julio de 2018 https://www.inegi.org.mx/temas/usosuelo/default.html_Descargas,

Instituto Nacional de Estadística y Geografía [Inegi]. (2017b. Anuario Estadístico y Geográfico del Estado de Puebla 2017. Gobierno del estado de Puebla. https://www.datatur.sectur.gob.mx/ITxEF_Docs/PUE_ANUARIO_ PDF.pdf

Instituto Nacional de Estadística y Geografía [Inegi]. (2018). Banco de Indicadores: Agricultura, ganadería, y pesca, Agricultura: Valor de la producción forestal maderable. Recuperado el 30 septiembre de 2018 https://www.inegi.o rg.mx/app/indicadores/?t=018000200020\&ag=00_tabMCcollapse-Indicadores

Llanos, L. (2010). El concepto del territorio y la investigación en las ciencias sociales. Agricultura, sociedad y desarrollo, 7(3), 207-220. http://www.scielo.org.mx/scielo.php?script=sci_arttext\&pid=SI870-5472201000030000I

Mas, J., Reyes, J. y Pérez, A. (2003). Evaluación de la confiabilidad temática de mapas o de imágenes clasificadas: una revisión. Investigaciones Geográficas, Boletín del Instituto de Geografía, (51), 53-72. http://www.scielo.org.mx/ pdf/igeo/n5i/n5ra5.pdf

McKean. M. A. y Ostrom, E. (1995). Common property regimes in the forest: Just a relic from the past? FAO Corporate Document Repository. https://dlc.dlib.indiana.edu/dlc/bitstream/handle/10535/3777/Common_property_ regimes_in_the_forest.pdf? sequence=I\&isAllowed=y

Mota Díaz, L., y Sandoval Forero, E. (2006). El rol del capital social en los procesos de desarrollo local. Límites y alcance en grupos indígenas. Economía, Sociedad y Territorio, V(20), 781-819. https://www.redalyc.org/pdf/I II/III02005.pdf

Ocampo R. (2014 Programa actual de la contabilidad ambiental-económica en México. Inegi. https://seea.un.org/sites/s eea.un.org/files/Presentations/Inception_Mission/Mexico/I.I_avancesecosistemas_130617_inegi.pdf

Organización de las Naciones Unidas [ONU]. (2012. Sistema de Contabilidad Ambiental y Económica. ONU, FMI, FAO, OCDE, BM. https://unstats.un.org/unsd/envaccounting/seearev/CF_trans/S_march2or4.pdf 
Quijano, A. (1992). Colonialidad y modernidad/racionalidad. Perú Indígena, I3(29), II-20.

Raffestin, C. (20II). Por una geografía del poder. El Colegio de Michoacán.

Registro Agrario Nacional [RAN]. (2012). Propiedad Social, fundamental para el desarrollo de México. Gobierno de México. Recuperado el 9 de febrero de 2016 de http://www.ran.gob.mx/ran/index.php/sala-de-prensa/hist orico/1342-propiedad-social-fundamental-para-el-desarrollo-de-mexico

Registro Agrario Nacional [RAN]. (2018). Padrón e Historial de Núcleos Agrarios (Pbina). Recuperado el 5 de septiembre de 2018 de http://www.ran.gob.mx/ran/index.php/sistemas-de-consulta/phina,

Rivera, J. (2006). El papel de la geografía en el estudio de la relación sociedad-naturaleza. Revista Luna Azul, (23), 23-27. https://www.redalyc.org/pdf/3217/321727225005.pdf

Salinas, E., González, J. M., León, A. y Rodríguez, F. (2017). La actividad forestal en el desarrollo económico de Chignahuapan, Puebla. Región y sociedad, 29(69), 185-218. https://doi.org/10.22198/rys.2017.69.a270

Secretaria de Medio Ambiente y Recursos Naturales [Semarnat]. (2018). Base de datos estadísticos del Sistema Nacional de Información Ambiental y Recursos Naturales. Recuperado el io de septiembre de 2018 de http://dgeiawf.semarnat.gob.mx:8080/ibi_apps/WFServlet?IBIF_ex=Dz_RFORESTAo4_or\%26IBIC_u ser=dgeia_mce\%26IBIC_pass=dgeia_mce\&NOMBREANIO=*\&NOMBREENTIDAD=Puebla

Valdés, O. y Negreros, P. (2010). El manejo forestal comunitario en México. México Forestal,

\section{Anexos}

\begin{tabular}{|c|c|c|c|c|}
\hline $\mathbf{N} .^{\circ}$ & $\begin{array}{l}\text { Clave } \\
\text { Inegi }\end{array}$ & Descripción & Clave CON & Descripción \\
\hline 1 & $\mathrm{HA}$ & Agricultura de humedad anual & \multirow{13}{*}{ AGR_AN } & \multirow{13}{*}{ Agricultura anual } \\
\hline 2 & HAP & Agricultura de humedad anual permanente & & \\
\hline 3 & HAS & Agricultura de humedad anual y semipermanente & & \\
\hline 4 & RA & Agricultura de riego anual & & \\
\hline 5 & RAP & Agricultura de riego anual y permanente & & \\
\hline 6 & RAS & Agricultura de riego anual y semipermanente & & \\
\hline 7 & RS & Agricultura de riego semipermanente & & \\
\hline 8 & RSP & Agricultura de riego semipermanente y permanente & & \\
\hline 9 & TA & Agricultura de temporal anual & & \\
\hline 10 & TAP & Agricultura de temporal anual y permanente & & \\
\hline 11 & TAS & Agricultura de temporal anual y semipermanente & & \\
\hline 12 & TS & Agricultura de temporal semipermanente & & \\
\hline 13 & TSP & Agricultura de temporal semipermanente y permanente & & \\
\hline 14 & $\mathrm{HP}$ & Agricultura de humedad permanente & \multirow{5}{*}{ AGR_PER } & \multirow{5}{*}{$\begin{array}{l}\text { Agricultura } \\
\text { permanente }\end{array}$} \\
\hline 15 & HS & Agricultura semipermanente & & \\
\hline 16 & HSP & Agricultura de humedad permanente y semipermanente & & \\
\hline 17 & $\mathrm{RP}$ & Agricultura de riego permanente & & \\
\hline 18 & TP & Agricultura de temporal permanente & & \\
\hline 19 & $\mathrm{AH}$ & Asentamientos humanos & \multirow{2}{*}{$\mathrm{AH}$} & \multirow{2}{*}{$\begin{array}{l}\text { Asentamientos } \\
\text { humanos }\end{array}$} \\
\hline 20 & $\mathrm{ZU}$ & Zona urbana & & \\
\hline 21 & BA & Bosque de oyamel & \multirow{12}{*}{$\mathrm{BCO} / \mathrm{P}$} & \multirow{12}{*}{$\begin{array}{c}\text { Bosque de coniferas } \\
\text { primario }\end{array}$} \\
\hline 22 & BB & Bosque de cedro & & \\
\hline 23 & $\mathrm{BJ}$ & Bosque de táscate & & \\
\hline 24 & BP & Bosque de pino & & \\
\hline 25 & BPQ & Bosque de pino-encino & & \\
\hline 26 & BS & Bosque de ayarin & & \\
\hline 27 & VSA/BA & Vegetación secundaria arbórea de bosque de oyamel & & \\
\hline 28 & $\mathrm{VSA} / \mathrm{BB}$ & Vegetación secundaria arbustiva de bosque de cedro & & \\
\hline 29 & VSA/BJ & Vegetación secundaria arbustiva de bosque de táscate & & \\
\hline 30 & VSA/BP & Vegetación secundaria arbustiva de bosque de pino & & \\
\hline 31 & VSA/BPQ & Vegetación secundaria arbustiva de bosque de pino-encino & & \\
\hline 32 & VSA/BS & Vegetación secundaria arbórea de bosque de ayarin & & \\
\hline
\end{tabular}

\section{ANEXO 1}

Tabla de equivalencias de las Clasificaciones INEgi-Conafor Fuente: Elaboración PRopia con base En ONU (2012), Conafor (2009), Inegi (2014). 


\begin{tabular}{|c|c|c|c|c|}
\hline 33 & $\mathrm{VSa} / \mathrm{BA}$ & Vegetación secundaria arbustiva de bosque de oyamel & \multirow{12}{*}{$\mathrm{BCO} / \mathrm{S}$} & \multirow{12}{*}{$\begin{array}{c}\text { Bosque de coniferas } \\
\text { secundario }\end{array}$} \\
\hline 34 & $\mathrm{VSa} / \mathrm{BB}$ & Vegetación secundaria arbustiva de bosque de cedro & & \\
\hline 35 & $\mathrm{VSa} / \mathrm{BJ}$ & Vegetación secundaria arbustiva de bosque de táscate & & \\
\hline 36 & $\mathrm{VSa} / \mathrm{BP}$ & Vegetación secundaria arbustiva de bosque de pino & & \\
\hline 37 & $\mathrm{VSa} / \mathrm{BPQ}$ & Vegetación secundaria arbustiva de bosque de pino-encino & & \\
\hline 38 & $\mathrm{VSa} / \mathrm{BS}$ & Vegetación secundaria arbórea de bosque de ayarin & & \\
\hline 39 & $\mathrm{VSa} / \mathrm{MJ}$ & Arbustiva de matorral de coniferas & & \\
\hline 40 & $\mathrm{VSh} / \mathrm{BA}$ & Herbácea de bosque de oyamel & & \\
\hline 41 & $\mathrm{VSh} / \mathrm{BJ}$ & Vegetación secundaria herbácea de bosque de táscate & & \\
\hline 42 & $\mathrm{VSh} / \mathrm{BP}$ & Vegetación secundaria herbácea de bosque de pino & & \\
\hline 43 & $\mathrm{VSh} / \mathrm{BPQ}$ & Vegetación secundaria herbácea de bosque de pino-encino & & \\
\hline 44 & $\mathrm{VSh} / \mathrm{MJ}$ & Vegetación secundaria herbácea de matorral de coniferas & & \\
\hline 45 & BQ & Bosque de encino & \multirow{4}{*}{$\mathrm{BE} / \mathrm{P}$} & \multirow{4}{*}{$\begin{array}{l}\text { Bosque de encino } \\
\text { primario }\end{array}$} \\
\hline 46 & $\mathrm{BQP}$ & Bosque de encino-pino & & \\
\hline 47 & $\mathrm{VSA} / \mathrm{BQ}$ & Vegetación secundaria arbustiva de bosque de encino & & \\
\hline 48 & VSA/BQP & Vegetación secundaria arbustiva de bosque de encino-pino & & \\
\hline 49 & $\mathrm{VSa} / \mathrm{BQ}$ & Vegetación secundaria arbustiva de bosque de encino & \multirow{4}{*}{$\mathrm{BE} / \mathrm{S}$} & \multirow{4}{*}{$\begin{array}{l}\text { Bosque de encino } \\
\text { secundario }\end{array}$} \\
\hline 50 & $\mathrm{VSa} / \mathrm{BQP}$ & Vegetación secundaria arbustiva de bosque de encino-pino & & \\
\hline 51 & $\mathrm{VSh} / \mathrm{BQ}$ & Vegetación secundaria herbácea de bosque de encino & & \\
\hline 52 & VSh/BQP & Vegetación secundaria herbácea de bosque encino-pino & & \\
\hline 53 & $\mathrm{BM}$ & Bosque mesófilo de montaña & \multirow{2}{*}{$\mathrm{BM} / \mathrm{P}$} & \multirow{2}{*}{$\begin{array}{c}\text { Bosque mesófilo de } \\
\text { montaña primario }\end{array}$} \\
\hline 54 & VSA/BM & Vegetación secundaria arbustiva de bosque mesófilo de montaña & & \\
\hline 55 & $\mathrm{VSa} / \mathrm{BM}$ & Vegetación secundaria arbustiva de bosque mesófilo de montaña & \multirow{2}{*}{$\mathrm{BM} / \mathrm{S}$} & \multirow{2}{*}{$\begin{array}{l}\text { Bosque mesófilo de } \\
\text { montaña secundario }\end{array}$} \\
\hline 56 & $\mathrm{VSh} / \mathrm{BM}$ & Vegetación secundaria herbácea de bosque de montaña & & \\
\hline 57 & $\mathrm{H} 2 \mathrm{O}$ & Cuerpos de agua continentales & $\mathrm{H} 2 \mathrm{O}$ & Cuerpos de agua \\
\hline 58 & MDM & Matorral desértico micrófilo & \multirow{5}{*}{$\mathrm{MXnL} / \mathrm{P}$} & \multirow{5}{*}{$\begin{array}{c}\text { Matorral xerófilo no } \\
\text { leñoso primario }\end{array}$} \\
\hline 59 & MDR & Matorral desértico rosetófilo & & \\
\hline 60 & VD & Vegetación de desiertos arenosos & & \\
\hline 61 & $\mathrm{VH}$ & Vegetación halófila xerófila & & \\
\hline 62 & VY & Vegetación gipsófila & & \\
\hline 63 & $\mathrm{ADV}$ & Desprovisto de vegetación & \multirow{2}{*}{ OT } & \multirow{2}{*}{ Otras tierras } \\
\hline 64 & DV & Sin vegetación aparente & & \\
\hline 65 & PC & Pastizal cultivado & \multirow{12}{*}{$\mathrm{P}$} & \multirow{12}{*}{ Pastizal } \\
\hline 66 & $\mathrm{PH}$ & Pastizal halófilo & & \\
\hline 67 & PI & Pastizal inducido & & \\
\hline 68 & PN & Pastizal natural & & \\
\hline 69 & PY & Pastizal gipsofilo & & \\
\hline 70 & VS & Sabana & & \\
\hline 71 & $\mathrm{VSa} / \mathrm{PH}$ & Vegetación secundaria arbustiva de pastizal halófilo & & \\
\hline 72 & $\mathrm{VSa} / \mathrm{PN}$ & Vegetación secundaria arbustiva de pastizal natural & & \\
\hline 73 & $\mathrm{VSa} / \mathrm{PY}$ & Vegetación secundaria arbustiva de pastizal gipsófilo & & \\
\hline 74 & $\mathrm{VSh} / \mathrm{PN}$ & Vegetación secundaria herbácea de pastizal natural & & \\
\hline 75 & VSI & Sabanoide & & \\
\hline 76 & VW & Pradera de alta montaña & & \\
\hline
\end{tabular}

ANEXO l.

Tabla de equivalencias de las Clasificaciones INEgi-Conafor

Fuente: elaboración PRopia con base En ONU (2012), Conafor (2009), Inegi (2014).

\section{Notas}

* Artículo de investigación. Agradezco al Consejo Nacional de Ciencia y Tecnología.

\section{BY}

